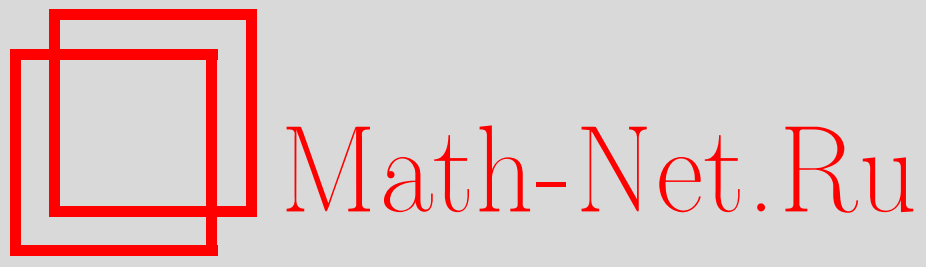

Р. Урибе-Варгас, Об устойчивости бифуркационных диаграмм исчезающих уплощений, Функи. анализ и его прил., 2003, том 37, выпуск 3, 88-94

DOI: https://doi.org/10.4213/faa163

Использование Общероссийского математического портала MathNet.Ru подразумевает, что вы прочитали и согласны с пользовательским соглашением

http://www. mathnet.ru/rus/agreement

Параметры загрузки:

IP : 54.162 .27 .143

26 апреля 2023 г., 13:19:21

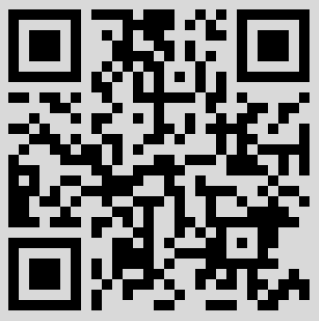


5. Гипотеза Шёнберга. Следующая теорема сформулирована Шёнбергом [13] в качестве гипотезы.

Теорема 12. Пусть $\left\{\lambda_{j}\right\}_{1}^{m}-$ корни полинома $q(\in \mathbb{C}[z])$ степени $m, a\left\{\mu_{j}\right\}_{1}^{m-1}$ - корни его производной. Если $\sum_{j=1}^{m} \lambda_{j}=0$, то

$$
m \sum_{j=1}^{m-1}\left|\mu_{j}\right|^{2} \leqslant(m-2) \sum_{j=1}^{m}\left|\lambda_{j}\right|^{2},
$$

причем равенство имеет место в точности тогда, когда числа $\left\{\lambda_{j}\right\}_{1}^{m}$ лежат на одной прямой.

Поясним идею доказательства. Пусть $A:=U \Lambda U^{*}$, где $\Lambda:=\operatorname{diag}\left(\lambda_{1}, \ldots, \lambda_{m}\right)$, $U=m^{-1 / 2}\left(\varepsilon^{k(j-1)}\right)_{k, j=1}^{m}$ и $\varepsilon=e^{2 \pi i / m}$. Можно показать, что матрица $A$ дает решение обратной задачи для наборов $\left\{\lambda_{j}\right\}_{1}^{m}$ и $\left\{\mu_{k}\right\}_{1}^{m-1}$, т. е. $\sigma(A)=\left\{\lambda_{j}\right\}_{1}^{m}$ и $\sigma\left(A_{m-1}\right)=\left\{\mu_{k}\right\}_{1}^{m-1}$. Легко видеть, что $A=m^{-1}\left(r\left(\varepsilon^{k-j}\right)\right)_{k, j=1}^{m}$, где $r(z):=$ $\sum_{j=1}^{m} \lambda_{j} z^{j-1}$. Отсюда выводится тождество $m\left\|A_{m-1}\right\|_{2}^{2}=(m-2)\|A\|_{2}^{2}$, в котором $\|A\|_{2}:=m^{-1}\left(\sum_{k, j}\left|r\left(\varepsilon^{k-j}\right)\right|^{2}\right)^{1 / 2}-$ фробениусова норма матрицы. Осталось заметить, что $m \sum_{j=1}^{m-1}\left|\mu_{j}\right|^{2} \leqslant m\left\|A_{m-1}\right\|_{2}^{2}=(m-2)\|A\|_{2}^{2}=(m-2) \sum_{j=1}^{m}\left|\lambda_{j}\right|^{2}$.

Благодарность. Я глубоко признателен $\Phi$. В. Петрову за ценные замечания, позволившие значительно улучшить текст статьи, а также за информацию о работах [1] и [12].

\title{
ЛитеРАТУРА
}

1. de Bruijn N. G., Springer T. A. Indag. Math., 9, 264-270 (1947). 2. Маламуд M. М. Укр. матем. ж., 44, №12, 1658-1688 (1992). 3. Malamud S. M. Linear Algebra Appl., 322, 19-41 (2001). 4. Маламуд С. М. Матем. заметки, 69, № 4, 633-637 (2001). 5. Маркус А. С. УМН, 19, вып. 4 (118), 93-123 (1965). 6. Маркус М., Минк Х. Обзор по теории матриц и матричных неравенств. Наука, M., 1972. 7. Marshall A. W., Olkin I. Inequalities: Theory of Majorization and Its applications. Academic Press, 1979. 8. Полиа Г., Сеге Г. Задачи и теоремы из анализа. Наука, М., 1978. 9. Харди Г., Литтлвуд Дж., Пойа Г. Неравенства. ИЛ, М., 1948. 10. Horn R., Johnson Ch. Matrix Analysis, Cambridge., 1986. 11. Pawlowski P. Trans. Amer. Math. Soc., 350, No. 11, 4461-4472 (1998). 12. Schmeisser G. In: Approximation Theory: A volume dedicated to Blagovest Sendov, DARBA, Sofia, 2002, pp. 353-369. 13. Schoenberg I. Amer. Math. Monthly, 93, 8-13 (1986).

Eidgenössische Technische Hochschule (ETH), Zürich e-mail: semka@math.ethz.ch

Поступило в редакцию 1 октября 2002 г.

УДК 514.75

\section{Об устойчивости бифуркационных диаграмм исчезающих уплощений}

\author{
(C) 2003. Р. УРИБЕ-ВАРГАС
}

1. Введение. Недавно В. И. Арнольд, исследуя особенности малых возмущений сильно вырожденных лагранжевых и лежандровых отображений (исчезающие особенности) получил различные модификации классической теоремы о че- 
тырех вершинах выпуклой плоской кривой (см. [2-4], а также $[6,7])$. В частности, были найдены новые оценки снизу числа точек уплощения пространственных кривых [2].

В этой заметке решена одна из задач В. И. Арнольда из этой области [5].

Точка пространственной кривой, в которой кручение равно нулю (первые три производные кривой линейно зависимы) называется точкой уплощения. Вырожденная точка уплощения - это точка кратного нуля кручения.

Нас интересуют точки уплощения семейства (исчезающих) пространственных кривых $\gamma(f, c): \mathbb{S}^{1} \rightarrow \mathbb{R}^{3}=\{x, y, w\}$, заданных как пересечения гладкой поверхности $w=f(x, y)$ в $\mathbb{R}^{3}$, являющейся графиком функции $f: \mathbb{R}^{2} \rightarrow \mathbb{R}$, с семейством соосных цилиндров $x^{2}+y^{2}=c$, радиус $r=\sqrt{c}$ которых стремится к нулю. Вычитание аффинной функции из $f$ не меняет проекции точек уплощения; поэтому можно всегда считать, что в нуле $f$ имеет критическую точку.

Если второй дифференциал $d^{2} f_{0}$ в нуле не является умбилическим (т.е. не пропорционален $\left.d x^{2}+d y^{2}\right)$, то для достаточно малых $c$ кривая $\gamma(f, c)$ имеет в точности четыре невырожденные точки уплощения.

Подмножество умбилических квадратичных форм от двух переменных имеет коразмерность два в трехмерном пространстве всех квадратичных форм от двух переменных. Поэтому умбилическое вырождение дифференциала $d^{2} f_{0}$ встречается только в семействах функций общего положения, зависящих от не менее чем двух параметров.

Бифуркациионая диаграмла $B(F)$ семейства функций $F: \mathbb{R}^{2} \times \Lambda \rightarrow \mathbb{R}$, зависящего от параметров $\lambda \in \Lambda=\mathbb{R}^{k}$, представляет собой множество пар $(\lambda, c) \in$ $\Lambda \times \mathbb{R}$, таких, что кривая $\gamma(F(\cdot, \lambda), c)$ имеет вырожденное уплощение.

Мы будем рассматривать особенности бифуркационной диаграммы (в точке $c=0)$ двухпараметрического семейства общего положения, являющегося деформацией функции $f=\left.F\right|_{\lambda=0}$ с умбилической критической точкой в нуле, с точностью до так называемой полярной эквивалентности семейств функций, которая сохраняет дифференцируемый тип бифуркационной диаграммы.

Три-струя такого семейства (с параметрами $a, b)$ эквивалентна струе $f_{0}=$ $x^{3}-3 x y^{2}+2 b(x y)+a\left(x^{2}-y^{2}\right)$ полиномиального семейства, бифуркационная диаграмма которого представляет собой параболическую поверхность (чашу) с шестью ребрами возврата, сходящимися в нуле (см. рис. 1).

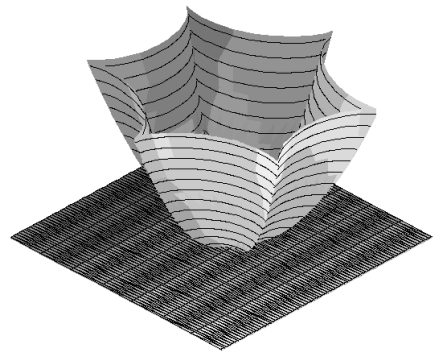

Рис. 1

Сечения этой поверхности координатным плоскостями $c=$ const являются гипоциклоидами с 6 точками возврата (траекториями точки окружности радиуса 
$r=\sqrt{c}$, катящейся без проскальзывания по внутренней стороне неподвижной окружности радиуса $6 r)$.

Бифуркационная диаграмма всякого продолжения семейства $f_{0}$ при добавлении членов более высокого порядка приближается к бифуркационной диаграмме семейства $f_{0}$ при $r \rightarrow 0$ (предложение 2 ). Параметры точек внутри чаши отвечают кривым с 6 простыми уплощениями, точкам вне чаши отвечают кривые с 4 точками уплощения. Регулярные точки самой поверхности отвечают кривым с двойным уплощениям, а точки полукубических ребер - кривым с тройными уплощениями.

Основным результатом заметки (теорема 1) является доказательство того, что с точностью до полярной эквивалентности умбилические семейства класса $C^{\infty}$ общего положения имеют единственный модуль (непрерывный инвариант орбиты). Используется техника, близкая к работе [1].

Автор благодарен В. И. Арнольду за постановку задачи, а также В. М. Закалюкину и М. Э. Казаряну за помощь.

2. Определения и результаты. Обозначим через $L$ производную вдоль гамильтонова векторного поля $V_{h}=-y \frac{\partial}{\partial x}+x \frac{\partial}{\partial y}$ на $\mathbb{R}^{2}$, касательного к окружностям $S_{c}: x^{2}+y^{2}=c$.

ПрЕДЛОЖЕНИЕ 1. Точки уплощения кривой $\gamma(f, c), c>0$, являются нулями ограничения функциии $L\left(L^{2}+1\right) f$ на $S_{c}$.

Доказательство состоит в непосредственном вычислении формы объема на первых трех производных кривой, параметризованной полярным углом $\varphi$. В полярных координатах оператор $L$ имеет вид $\frac{\partial}{\partial \varphi}$.

Обозначим через $\hat{f}=L\left(L^{2}+1\right) f$ образ функции $f$ под действием оператора $L\left(L^{2}+1\right)$. Вырожденные уплощения отвечают общим нулям $\hat{f}=0$ и $L \hat{f}=0$. Следовательно, семейства функций, имеющие одинаковый образ при действии $L\left(L^{2}+1\right)$, имеют одинаковые бифуркационные диаграммы.

Для заданного семейства $F: \mathbb{R}^{2} \times \mathbb{R}^{k} \rightarrow \mathbb{R}$ функций, зависящего от параметров $\lambda \in \mathbb{R}^{k}$, семейство $\widehat{F}=L\left(L^{2}+1\right) F$ назовем производящим семейством бифуркационной диаграммы $B(F)=\{(\lambda, c) \mid \exists(x, y): \widehat{F}=L \widehat{F}=0, h=0\}$, где $h(x, y, c)=x^{2}+y^{2}-c$. Семейство отображений $H_{\widehat{F}}: \mathbb{R}^{2} \times \mathbb{R}^{k} \times \mathbb{R}_{+} \rightarrow \mathbb{R}^{2}$, $H_{\widehat{F}}:(x, y, \lambda, c) \mapsto(\widehat{F}, h)$, зависящее от дополнительного параметра $c \in \mathbb{R}, c \geqslant 0$, назовем определяющим отображением.

ОПредЕлЕния. Два семейства $F_{1}, F_{2}: \mathbb{R}^{2} \times \mathbb{R}^{k} \rightarrow \mathbb{R}$ называются ичилиндрически әквивалентными, если их определяющие отображения контактно эквивалентны, т. е. если существуют невырожденная $2 \times 2$-матрица $M$, элементы которой являются гладкими функциями от $(x, y, \lambda, c)$, и диффеоморфизм $\Theta: \mathbb{R}^{2} \times$ $\mathbb{R}^{k} \times \mathbb{R} \rightarrow \mathbb{R}^{2} \times \mathbb{R}^{k} \times \mathbb{R}$, сохраняющий границу $c=0$ и сохраняющий расслоение $\pi:(x, y, \lambda, c) \mapsto(\lambda, c)$ над параметрами (т. е. $\pi \circ \Theta=\Theta^{\prime} \circ \pi$ для некоторого диффеоморфизма $\Theta^{\prime}: \mathbb{R}^{k} \times \mathbb{R} \rightarrow \mathbb{R}^{k} \times \mathbb{R}$ пространства параметров), такие, что:

$$
H_{F_{2}} \circ \Theta=M \cdot H_{F_{1}} \text {. }
$$

Два семейства $F_{1}, F_{2}: \mathbb{R}^{2} \times \mathbb{R}^{k} \rightarrow \mathbb{R}$ называются полярно эквивалентными, если их образы $\widehat{F}_{1}, \widehat{F}_{2}$ цилиндрически эквивалентны. 
ЗАмЕчАНИЯ. 1. Очевидно, что цилиндрически эквивалентные производящие семейства (а, следовательно, и полярно эквивалентные семейства) задают диффеоморфные бифуркационные диаграммы.

2. Орбиты цилиндрической эквивалентности семейств $F+\phi\left(x^{2}+y^{2}-c\right)$ по $x$, $y, \lambda, c$ для различных функций $\phi$ совпадают. Поэтому в дальнейшем мы будем отождествлять между собой семейства функций от $x, y$ с параметрами $a, b, c$, отличающиеся на функцию из идеала, порожденного уравнением $x^{2}+y^{2}-c=0$.

В комплекснозначных координатах $z=x+i y, \bar{z}=x-i y$ на $\mathbb{R}^{2}$ оператор $L$ принимает вид $L=i\left(z \frac{\partial}{\partial z}-\bar{z} \frac{\partial}{\partial \bar{z}}\right)$. Всякий моном $z^{m} \bar{z}^{n}$ является собственной функцией оператора $L\left(L^{2}+1\right)$ с собственным значением $\left((n-m)^{2}-1\right)(n-m) i$. В частности, мономы $z^{m} \bar{z}^{m}=\left(x^{2}+y^{2}\right)^{m}, z^{m+1} \bar{z}^{m}, z^{m} \bar{z}^{m+1}$ принадлежат ядру оператора $L\left(L^{2}+1\right)$.

Алгебру $\mathscr{O}_{X}$ ростков в нуле (вещественных) гладких функций от $x, y$ (эквивалентно, от $z, \bar{z})$ и алгебру $\mathscr{O}_{\Lambda}$ ростков в нуле функций от параметров $a, b, c$ будем отождествлять с их образами при естественном вложении в алгебру $\mathscr{O}_{X, \Lambda}$ ростков в нуле функций от всех переменных $x, y, a, b, c$. Обозначим через $\mathscr{M}_{\alpha}$ максимальный идеал в $\mathscr{O}_{\alpha}$, а через $K_{\alpha}$ и $I_{\alpha}$ соответственно ядро и образ оператора $L\left(L^{2}+1\right)$, действующего на пространстве $\mathscr{O}_{\alpha}$ (здесь « $\alpha »$ заменяет один из символов $X, \Lambda$ или $X \Lambda)$.

Пространство ростков 3 -струй функций от $z, \bar{z}$ порождается мономами $z^{3}, \bar{z}^{3}$, $z^{2}, \bar{z}^{2}$, а также мономами $1, z, \bar{z}, z \bar{z}, z^{2} \bar{z}, z \bar{z}^{2}$ из $K_{X}$. Следовательно, с помощью подходящих гомотетии, поворота осей $x, y$ и замены параметров (задающих полярную эквивалентность) 3 -струю по $z, \bar{z}$ (с коэффициентами из $\mathscr{O}_{\Lambda}$ ) семейства общего положения $F(z, \bar{z}, a, b)$ в умбилической точке можно привести к 3 -струе стандартного семейства $f_{0}$, для которого

$$
\hat{f}_{0}=\frac{1}{2}\left(z^{3}+\bar{z}^{3}+A z^{2}+\bar{A} \bar{z}^{2}\right)=x^{3}-3 x y^{2}+a\left(x^{2}-y^{2}\right)+2 b x y,
$$

где $A=a+i b$.

ПрЕДЛОЖЕНИЕ 2. (i) БифурКационная диаграмма $B\left(f_{0}\right)$ семейства $f_{0}(x, y, a, b)$ состоит из плоскости $c=0$ и поверхности («гипоциклоидной иаши») $\Sigma_{f_{0}} \hookrightarrow$ $\mathbb{C} \times \mathbb{R}=\mathbb{R}^{3}$, заданной параметризацией $P_{f_{0}}:(\varphi, c) \mapsto\left(-2 c^{1 / 2}\left(5 e^{-i \varphi}+e^{5 i \varphi}\right), c\right)$ для $c \geqslant 0$ (см. рис. 1).

(ii) Сечения $(c=$ const $>0)$ бифуркационной диаграммы $B(F)$ для деформациии $F$ функции с 3-струей $f_{0}$ диффеоморфнь гипоичклоидам с 6 точками возврата и имеют параметризаичюю $P_{F}:(\varphi, c) \mapsto\left(-2 c^{1 / 2}\left(5 e^{-i \varphi}+e^{5 i \varphi}\right)+\right.$ $O(c), c)$, отличающуюся членом порядка $O(c)(c \rightarrow 0)$ от параметризации семейства $f_{0}$.

ДокАЗАТЕЛьство. 1. Положив $z=r e^{i \varphi}$, запишем уравнения, задающие бифуркационную диаграмму $B\left(f_{0}\right)$, в виде

$$
\operatorname{Re}\left(4 i r^{3} e^{-3 i \varphi}+A i r^{2} e^{-2 i \varphi}\right)=0 \quad \text { и } \quad \operatorname{Re}\left(6 r^{3} e^{-3 i \varphi}+A r^{2} e^{-2 i \varphi}\right)=0 .
$$

Отсюда следует, что либо $c=r^{2}=0$, либо $A(\varphi, c)=-2 c^{1 / 2}\left(5 e^{-i \varphi}+e^{5 i \varphi}\right)$.

2. Уравнения, задающие $B(F)$, отличаются от уравнений, задающих $B\left(f_{0}\right)$, членами порядка $c^{2}$. При разрешении их относительно $A$ поправочный член имеет порядок $O(c)$. Отображение $\left.P_{f_{0}}\right|_{c=\text { const }}: S_{c} \rightarrow R^{2}$ является устойчивым лежандровым отображением. Следовательно, для достаточно малых $c$ лежандрово 
отображение $\left.P_{F}\right|_{c=\text { const }}$ будет лежандрово эквивалентно $\left.P_{f_{0}}\right|_{c=\text { const }}$, а их фронты (сечения бифуркационных диаграмм) диффеоморфны.

Теорема 1. Семейство $F=f_{0}+\varphi(x, y, a, b)$ с нулевой 3 -струей $\varphi$ полярно эквивалентно или $f_{0}+\cos \alpha\left(x^{5}-10 x^{3} y^{2}+5 x y^{4}\right)+\sin \alpha\left(5 x^{4} y-10 x^{2} y^{3}+y^{5}\right)$ nри некотором значении $\alpha \in \mathbb{R}(\bmod 2 \pi)$, или $f_{0}+x^{6}-15 x^{4} y^{2}+15 x^{2} y^{4}-y^{6}$, или $f_{0}$.

Таким образом, бифуркационная диаграмма семейства $F$ имеет не более одного модуля и диффеоморфна бифуркационной диаграмме семейства одной из перечисленных в теореме нормальных форм.

3. Доказательство теоремы 1. Будем следовать стандартному методу (используя подготовительную теорему Мальгранжа и гомотопический метод), чтобы привести цилиндрической эквивалентностью производящее семейство к нормальной форме, оставаясь при этом в образе $I_{X, \Lambda}$. Однако нам придется преодолеть трудности, связанные с тем. что образ $I_{X, \Lambda}$ является модулем только над кольцом $\mathscr{O}_{\Lambda}$, а не над кольцом $\mathscr{O}_{X, \Lambda}$.

Действие инфинитезимальных цилиндрических эквивалентностей на росток семейства $F \in \mathscr{O}_{X, \Lambda} \mathscr{M}_{X}$ задает касательное пространство $T F$ к орбите цилиндрической эквивалентности.

ЛЕмма 1. Касательное пространство ТF состоит из ростков функизй $\Phi \in \mathscr{O}_{X, \Lambda}$, имеющих разложение

$$
\begin{aligned}
\Phi=u_{1} F & +u_{2} L(F)+u_{3}(z \bar{z}-c) \\
& +u_{4}\left(\frac{\partial F}{\partial z} V_{\bar{z}}+\frac{\partial F}{\partial \bar{z}} V_{z}\right)+v_{1} \frac{\partial F}{\partial a}+v_{2} \frac{\partial F}{\partial b}+v_{3}\left(z \frac{\partial F}{\partial z}+\bar{z} \frac{\partial F}{\partial \bar{z}}\right)
\end{aligned}
$$

с некоторыми гладкими функциями $u_{i}=u_{i}(x, y, a, b, c), i=1, \ldots, 4, v_{j}=$ $v_{j}(a, b, c), j=1,2,3$, и функциями $V_{z}, V_{\bar{z}}$ от $x, y, a, b, c$, такими, ито $F=z V_{z}+\bar{z} V_{\bar{z}}$. Пространство $T_{F}$ является $\mathscr{O}_{\Lambda}$-модулем.

ДокАЗАтЕльство. Разложение (1) получается дифференцированием по $t \in \mathbb{R}$ в $t=0$ (векторного) равенства $H_{F+t \Phi}=M_{t} \cdot H_{F+t \Phi} \circ \Theta_{t}^{-1}$ и нахождением образующих модуля решений $g, \bar{g} \in \mathscr{O}_{X, \Lambda}, \rho \in \mathscr{O}_{\Lambda}$ уравнения для вторых компонент $0=\dot{m}_{2,1} F+\dot{m}_{2,2} h+\frac{\partial h}{\delta z} g+\frac{\partial h}{\delta \bar{z}} \bar{g}+\frac{\partial h}{\delta c} \rho$, где $\dot{m}_{2,1}, \dot{m}_{2,2} \in \mathscr{O}_{X, \Lambda}$.

Обозначим через $\mathscr{O}_{p}=\mathscr{O}_{X, \Lambda} \mathscr{M}_{X}^{p}$ пространство ростков $\varphi$, имеющих нулевую $(p-1)$-струю по $z, \bar{z}$. Пусть $T_{4} F=\mathscr{O}_{4} \cap T F$ и $N_{4} F=\mathscr{O}_{4} / T_{4} F-$ соответствующие касательное и нормальное подпространства. Квадратными скобками обозначим соответствующие пространства в алгебре формальных степенных рядов; например, формальное касательное подпространство $[T F] \subset\left[\mathscr{O}_{X, \Lambda}\right]$ задается разложением (1) для формальных рядов от $z, \bar{z}, a, b, c$.

Лемма 2. (i) Формальное нормальное пространство $\left[N_{4} \hat{f}_{0}\right]$ порождено над $\mathbb{R}$ классами ростков $z^{5}, \bar{z}^{5}, z^{6}+\bar{z}^{6}$, а также классом ростка $z^{2} \bar{z}^{2} \in K_{X}$. В частности, всякий моном из $\left[\mathscr{O}_{4}\right]$ суммарной степени $\geqslant 7$ по $z, \bar{z}, a, b, c$ принадлежит $\left[T f_{0}\right]$.

(ii) Предьдущее утверждение остается верным, если $\hat{f}_{0}$ заменить семейством $F=\hat{f}_{0}+\varphi$ с произвольным ростком $\varphi \in \mathscr{O}_{4}$.

ДокАЗАТЕльство. (i) Все мономы суммарной степени $\leqslant 7$ (при условии, что их степень по $z, \bar{z}$ не меньше 4$)$, за исключением перечисленных в лемме, можно разложить по формуле $(1)$, используя квазиоднородность $\hat{f}_{0}(z, \bar{z}, a, b$, имеют 
веса 1 , а $c$ имеет вес 2). Например, $z^{2}+\bar{z}^{2}=2 \frac{\partial \hat{f}_{0}}{\partial a}, i\left(z^{2}-\bar{z}^{2}\right)=2 \frac{\partial \hat{f}_{0}}{\partial b}, b z \bar{z}^{2}=$ $\left.a^{2} z^{2}+\frac{1}{5}(a b-c) \bar{z}^{2}\left(\bmod \hat{f}_{0}, L\left(\hat{f}_{0}\right)\right\}\right)$.

Непосредственные вычисления показывают, что однородные подпространства в $\left[N_{4} \hat{f}_{0}\right]$ степеней $4,5,6,7$ имеют соответственно размерности $1,2,1$ и 0 . Кроме того, подпространство в $\left[T_{4} \hat{f}_{0}\right]$, отвечающее разложениям с $u_{4}=0$, совпадает со всем пространством $\left[T_{4} \hat{f}_{0}\right]$. Поскольку $T\left[\hat{f}_{0}\right]$ является $\left[\mathscr{O}_{\Lambda}\right]$-модулем, остается только найти разложения для всякого монома $g$ степени $\geqslant 8$ по $z$ и $\bar{z}$. Поскольку $g$ делится либо на $z^{3}$, либо на $\bar{z}^{3}$, этот моном сравним с полиномом меньшей степени по $z$ и $\bar{z}$ по модулю идеала, порожденного $2 f_{0}=z^{3}+\bar{z}^{3}+A z^{2}+\bar{A} \bar{z}^{2}$ и $\frac{2}{3} L\left(f_{0}\right)=i\left(z^{3}-\bar{z}^{3}+2 / 3 A z^{2}-2 / 3 \bar{A} \bar{z}^{2}\right)$. После нескольких шагов степень понизится до 7, и, следовательно, искомое разложение существует.

(ii) C помощью введенной выше квазиоднородной фильтрации представим некоторый квазиоднородный росток $\psi_{m}$ степени $m \geqslant 4$ в виде суммы $\mathbb{R}$-линейной комбинации образующих пространства $\left[N_{4} \hat{f}_{0}\right]$ степени $m$ и ростка $\psi_{m}^{(0)} \in\left[T_{4} \hat{f}_{0}\right]$, допускающего разложение $(1)$ при $F=\hat{f}_{0}$ и квазиоднородных $u_{i}, v_{j}$ соответствующих степеней. Разложение $(1)$ с теми же $u_{i}, v_{j}$, но с функцией $F=\hat{f}_{0}+\varphi$ отличается от $\psi_{m}^{(0)}$ на поправочный член $\psi^{(1)}$ степени $>m$ и принадлежит $\left[T_{4} F\right]$. Таким образом, искомое формальное представление для всякого $\psi_{m}$ строится индуктивно. На каждом шаге к построенным формальным рядам добавляется разложение поправочного члена $\psi^{(l)}$. Новый поправочный член имеет бо́льшую степень.

Лемма 3. (i) Касательное пространство $T_{4} F$ для всякого $F=\hat{f}_{0}+\varphi$ при $\varphi \in \mathscr{O}_{4}$ содержит пересечение $\mathscr{O}_{4} \cap \mathscr{M}_{X, \Lambda}^{7}$.

(ii) Росток $F=\hat{f}_{0}+\varphi\left(\right.$ рри $\left.\varphi \in \mathscr{O}_{4}\right)$ является 7-определенным, т.е. он цилиндрически эквивалентен всякому ростку $\widetilde{F}=F+\tilde{\varphi}$ при $\tilde{\varphi} \in \mathscr{O}_{4} \cap \mathscr{M}_{X, \Lambda}^{7}$.

ДокАзАтельство. (i) Классы мономов $e_{1}=1, e_{2}=z, e_{3}=\bar{z}, e_{4}=z^{2}$, $e_{5}=\bar{z}^{2}$ образуют базис локальной алгебры $Q=\mathscr{O}_{x} / J$, где $J \in \mathscr{O}_{X}-$ идеал, порожденный ростками $\left.F\right|_{a, b, c=0}$ и $\left.L(F)\right|_{a, b, c=0}$. Из подготовительной теоремы Мальгранжа, примененной к отображению $(z, \bar{z}, a, b, c) \mapsto(F, L(F), a, b, c)$, вытекает, что классы этих же мономов $e_{i}, i=1, \ldots, 5$, порождают над $\mathscr{O}_{\Lambda}$ модуль $E=\mathscr{O}_{X, \Lambda} / J$, где $J=\mathscr{O}_{X, \Lambda}\{F, L(F)\} \subset T F$. Поскольку $J \subset \mathscr{M}_{X, \Lambda}^{3}$ и степени мономов $e_{i}$ меньше 3 , имеет место следующее включение: $\mathscr{M}_{X, \Lambda}^{3} \subset \mathscr{M}_{\Lambda} E+J$.

Если $g_{j}$ образуют конечную систему (однородных) образующих $\mathscr{O}_{X, \Lambda}$-модуля $B=\mathscr{O}_{4} \cap \mathscr{M}_{X, \Lambda}^{7}$, то $B \subset J+\mathscr{O}_{\Lambda}\left\{e_{i} g_{j}\right\}$ и $\mathscr{O}_{4} \cap \mathscr{M}_{X, \Lambda}^{10}=\mathscr{M}_{X, \Lambda}^{3} \cdot B \subset J+\mathscr{M}_{\Lambda}\left\{e_{i} g_{j}\right\}$.

Из утверждения (ii) леммы 2 следует, что всякий $e_{i} g_{j}$ принадлежит $T F$ с точностью до поправочного члена произвольно высокой степени, в частности, степени $\geqslant 10$. Теперь, используя включение $J \subset T F$, получаем, что $T F+\mathscr{O}_{\Lambda}\left\{e_{i} g_{j}\right\} \subset$ $T F+\mathscr{M}_{\Lambda}\left\{e_{i} g_{j}\right\}$. Наконец, из леммы Накаямы вытекает, что $\mathscr{O}_{\Lambda}\left\{e_{i} g_{j}\right\} \subset T F$, или, равносильно, $\mathscr{O}_{4} \cap \mathscr{M}_{X, \Lambda}^{7} \subset T F$, что и требовалось.

(ii) Утверждение (ii) является следствием предыдущего утверждения (i). Действительно, гомологическое уравнение, отвечающее гомотопии $t \in[0,1]$ семейств $\widetilde{F}_{t}=F+t \tilde{\varphi}$, имеет решение, поскольку $-\tilde{\varphi} \in T F_{t}$ при всяком $t$.

Для завершения доказательства осталось найти цилиндрическую эквивалентность, приводящую 6 -струю семейства $\widehat{F}=\hat{f}_{0}+\hat{\varphi}$ к указанной нормальной форме 
(мы рассматриваем струи функций от $z, \bar{z}$ с коэффициентами, гладко зависящими от параметров).

Следуя методу спектральной последовательности Арнольда, заметим сначала, что $j^{4}(T \widehat{F})=j^{4}\left(T \hat{f}_{0}\right)$. Поскольку $\hat{\varphi} \in I_{X \Lambda}$, то его 4 -струя не содержит монома $z^{2} \bar{z}^{2}$. Поэтому (лемма 2) $\hat{\varphi} \in j^{4}(T \widehat{F})$ и гомологическое уравнение цилиндрической эквивалентности для гомотопии $\widehat{F}_{t}=\hat{f}_{0}+t \hat{\varphi}$ имеет решение в пространстве 4-струй.

Таким образом, росток $\widehat{F}$ цилиндрически эквивалентен ростку $\widehat{F}_{(5)}=\hat{f}_{0}+\hat{\varphi}_{(5)}$, где $\hat{\varphi}_{(5)} \in \mathscr{O}_{5}$. Струи порядка 5 касательных пространств $T \widehat{F}$ и $T \hat{f}_{0}$ совпадают. Если $\hat{\varphi}_{(5)}$ имеет ненулевой класс $q=\alpha z^{5}+\bar{\alpha} \bar{z}^{5}$ при проекции на нормальное подпространство, то коэффициенты $\alpha$ можно нормировать так, чтобы $|\alpha|=1$, применяя гомотетию, заданную эйлеровой инфинитезимальной цилиндрической эквивалентностью $E$, которая строится по разложению (1) при $u_{1}=-3, u_{2}=$ $u_{3}=u_{4}=0, v_{1}=a, v_{2}=b, v_{3}=1$ (и которая принадлежит стационарной подалгебре для $\left.\hat{f}_{0}: E\left(\hat{f}_{0}\right)=0\right)$.

Поскольку $j^{5}\left(\hat{\varphi}_{(5)}-q\right) \in j^{5}\left(T \widehat{F}_{(5)}\right)$, семейство $\widehat{F}_{(5)}$ цилиндрически эквивалентно семейству $\widehat{F}_{(6)}=\hat{f}_{0}+q+\hat{\varphi}_{(6)}$ при некотором $\hat{\varphi}_{(6)} \in \mathscr{O}_{6}$. Вычисляя $j^{6}\left(T \widehat{F}_{(6)}\right)$ при $\alpha \neq 0$, получаем, что $z^{6}+\bar{z}^{6} \in \mathbb{R}\{A, \bar{A}\} E\left(\widehat{F}_{(6)}\right)+T\left(\hat{f}_{0}+q\right)$. В этом случае по лемме 26 -струя семейства $\widehat{F}_{(6)}$ цилиндрически эквивалентна $\hat{f}_{0}+q$.

В оставшемся случае, когда $\alpha=0,5$-струя имеет нормальную форму $\hat{f}_{0}$ и 6 -струя цилиндрически эквивалентна $\hat{f}_{0}+p\left(\bar{z}^{6}+z^{6}\right)$. Отличный от нуля коэффициент $p$ можно нормировать, используя $E$. Наконец, если $p=0$, то росток приводится к нормальной форме $\hat{f}_{0}$. По лемме 3 члены высших степеней можно отбросить. Теорема доказана.

\section{ЛИТЕРАТУРА}

1. Agrachev A. A., Charlot G., Gautier J. P., Zakalyukin V. M. C. R. Acad. Sci. Paris, 330, Sér. I, No. 6, 465-470 (2000). 2. Arnold V. I. Sur les propriétés des projections Lagrangiennes en géométrie symplectique des caustiques (Cahiers de Math. de la Decision, 9320 CEREMADE, 1993, 1-9). Rev. Mat. Univ. Complut. Madrid, 8, No. 1, 109-119 (1995). 3. Arnold V. I. Amer. Math. Soc. Transl., 171, 11-22 (1995). 4. Арнольд В. И. Функц. анализ и его прил., 32, вып. 2, 1-7 (1998). 5. Арнольд В. И. и др. Задача 1993-3 из книги Задачи Арнольда. Фазис, Москва, 2000. 6. Kazarian M. Nonlinear Version of Arnold's Theorem on Flattening Points. C. R. Acad. Sci. Paris, 323, Sér. I, No. 1, 63-68 (1996). 7. Uribe-Vargas R. C. R. Acad. Sci. Paris, 330, Sér. I, 1085-1090 (2000).

Université Paris 7, equipe Géométrie et Dynamique e-mail: uribe@math.jussieu.fr

Поступило в редакцию http://www.math.jussieu.fr/ uribe/

13 мая 2002 г. 